PROCEEDINGS OF THE

AMERICAN MATHEMATICAL SOCIETY

Volume 128, Number 10, Pages 2855-2862

S 0002-9939(00)05331-4

Article electronically published on April 28, 2000

\title{
ON REFLEXIVITY OF DIRECT SUMS
}

\author{
V. P. CAMILLO AND K. R. FULLER
}

(Communicated by Ken Goodearl)

\begin{abstract}
Necessary and sufficient conditions are presented to insure that the direct sum of two reflexive representations of a finite dimensional algebra is reflexive, and it is shown that for each such algebra, there is an integer $k$ such that the direct sum of $k$ copies of each of its representations is reflexive. Given a ring $\Delta$, our results are actually presented in the more general setting of $\Delta$-representations of a ring $R$.
\end{abstract}

Over the last thirty years, a significant amount of attention has been given to the problem of determining when an algebra $A$ of operators on a vector space $V$ (often a Hilbert space) over a field $K$ is reflexive in the sense that no larger algebra of operators on that space has the same lattice of invariant subspaces. (See, for example, 2 , 3], 6], 7], 12], 13, 14].) Of course, $A$ is an algebra of operators on a vector space $V$ if and only if $V$ is a faithful left $A$-module. Thus, to attack this problem from a more module theoretic point of view, based on notation of Halmos [14, the following notions were presented in [9]: For any $R-\Delta$-bimodule $M={ }_{R} M_{\Delta}$ one defines

$$
\operatorname{alglat}(M)=\left\{\alpha \in \operatorname{End}\left(M_{\Delta}\right) \mid \alpha L \subseteq L \text { for all }{ }_{R} L \leq{ }_{R} M\right\},
$$

and, letting $\lambda: R \rightarrow \operatorname{alglat}\left(M_{\Delta}\right)$ denote the canonical ring homomorphism, $M$ is called a reflexive bimodule (or $\Delta$-representation of $R$ ) if $\lambda$ is surjective. Thus the $K$-algebra of operators $A$ on $V$ is reflexive if and only if ${ }_{A} V_{K}$ is a reflexive bimodule (and then we simply say that the $A$-module $V$ is reflexive).

The problems we shall deal with here have their roots in the papers [5] and [6] of Deddens and Fillmore and [2] of Azoff. Stated in module theoretic terms, in the first pair of papers the question was posed and answered (affirmatively) of whether a direct sum of two finitely generated reflexive modules over an algebra generated by a single complex matrix is again reflexive; and Azoff showed that for each finite dimensional module over a $\mathbb{C}$-algebra, there is a positive integer $k$, depending on its dimension, such that the direct sum of $k$ copies of that module is reflexive. Other results in this vein can be found in 4 where Brenner and Butler showed that the direct sum of two copies of the regular representation of a finite dimensional algebra is reflexive, and in Habibi and Gustafson's [12] from which the same result follows for any faithful representation of a split serial algebra. (See [9], 10] and [1] for related and more general results.)

Our main objectives are to provide, in Theorem 1.2, a necessary and sufficient condition for a direct sum of two reflexive bimodules to be reflexive; and to show, in

Received by the editors September 10, 1998 and, in revised form, November 10, 1998.

1991 Mathematics Subject Classification. Primary 16D20, 16G99, 16P10; Secondary 47A15. 
Theorem 3.2 that whenever $R$ is a left artinian ring with composition length $c\left({ }_{R} R\right)$, there is a positive integer $k \leq c\left({ }_{R} R\right)+1$ such that the direct sum $M^{(k)}$ of $k$ copies of $M$ is reflexive for any bimodule ${ }_{R} M_{\Delta}$. Along the way we show how Theorem 1.2 can be employed to obtain simple proofs of some known results, and we examine a common generalization of generating and cogenerating called controlling that was introduced and employed in [9] and [10], showing in particular that if ${ }_{R} M$ is faithful and reflexive, then $M \oplus N$ is reflexive if and only if $M$ controls $N$.

\section{A characterization}

Unless otherwise specified, all modules under consideration will be left- $R$, right$\Delta$ bimodules for a fixed pair of rings $R$ and $\Delta$. If $R$ is an algebra over a field $K$, we shall assume that $\Delta=K$. If $\alpha \in \operatorname{End}\left(M_{\Delta}\right)$ and $\beta \in \operatorname{End}\left(N_{\Delta}\right)$, we shall write $(\alpha, \beta) \in \operatorname{End}(M \oplus N)$ for the direct sum map $(\alpha, \beta):(m, n) \mapsto(\alpha m, \beta n)$. Thus if $\gamma \in \operatorname{alglat}(M \oplus N)$, then $\gamma=\left(\left.\gamma\right|_{M},\left.\gamma\right|_{N}\right)$.

A common subquotient of a pair of left modules ${ }_{R} M$ and ${ }_{R} N$ is a module ${ }_{R} X$ that is isomorphic to a subquotient of both ${ }_{R} M$ and ${ }_{R} N$. This notion together with the following lemma allows us to determine just when the direct sum of a pair of reflexive modules is reflexive.

Lemma 1.1. If $r, s \in R$, the ordered pair $(r, s) \in \operatorname{alglat}(M \oplus N)$ if and only if $(r-s) X=0$, for every (equivalently, every cyclic) common subquotient of ${ }_{R} M$ and ${ }_{R} N$.

Proof. Note that $a \in R$ annihilates every common subquotient of $M$ and $N$ if and only it annihilates each of their cyclic common subquotients. According to Goursats' Lemma, $W$ is an $R$-submodule of $M \oplus N$, if and only if there are submodules

$$
M_{2} \leq M_{1} \leq M \text { and } N_{2} \leq N_{1} \leq N
$$

and an $R$-isomorphism

$$
f: M_{1} / M_{2} \rightarrow N_{1} / N_{2}
$$

such that

$$
W=\left\{\left(m_{1}, n_{1}\right) \in M_{1} \oplus N_{1} \mid f\left(m_{1}+M_{2}\right)=n_{1}+N_{2}\right\} .
$$

(Given $W$ and the orthogonal projections $\pi_{M}$ and $\pi_{N}$ for $M \oplus N$, one checks that $M_{1}=\pi_{M}(W), M_{2}=M \cap W, N_{1}=\pi_{N}(W)$, and $N_{2}=N \cap W$.)

$(\Rightarrow)$ If $\left(m_{1}, n_{1}\right) \in W$ and $\left(r m_{1}, s n_{1}\right) \in W$, then

$$
f\left(r m_{1}+M_{2}\right)=s n_{1}+N_{2}=s f\left(m_{1}+M_{2}\right)
$$

so, since $f$ is an $R$-isomorphism, $(r-s)\left(M_{1} / M_{2}\right)=0$.

$(\Leftarrow)$ Suppose $(r-s)\left(M_{1} / M_{2}\right)=0$, and $f: M_{1} / M_{2} \rightarrow N_{1} / N_{2}$ is an isomorphism and $\left(m_{1}, n_{1}\right) \in W$. Then

$$
f\left(r m_{1}+M_{2}\right)=r f\left(m_{1}+M_{2}\right)=r n_{1}+N_{2}=s n_{1}+N_{2}
$$

so $\left(r m_{1}, s n_{1}\right) \in W$.

Now we are able to provide the promised characterization in terms of annihilators of subquotients of $M$ and $N$. The left annihilator of a module $M$ is $\ell_{R}(M)=$ $\{r \in R \mid r M=0\}$. 
Theorem 1.2. Let $M$ and $N$ be reflexive, and let $\left\{X_{i} \mid i \in I\right\}$ represent one copy of each of the (cyclic) common subquotients of ${ }_{R} M$ and ${ }_{R} N$. Then $M \oplus N$ is reflexive if and only if

$$
\ell_{R}(M)+\ell_{R}(N)=\ell_{R}\left(\bigoplus_{i \in I} X_{i}\right)
$$

Proof. $(\Leftarrow)$ Let $\alpha=(\beta, \gamma) \in \operatorname{alglat}(M \oplus N)$. Then there are $r, s \in R$ with $\beta=r$ and $\gamma=s$, and by Lemma 1.1, $r-s \in \ell_{R}\left(\bigoplus_{i \in I} X_{i}\right)$. But then by hypothesis

$$
r-s=p-q \text { with } p \in \ell_{R}(M) \text { and } q \in \ell_{R}(N)
$$

so that, letting

$$
t=r-p=s-q,
$$

we have $\alpha=\lambda(t)$.

$(\Rightarrow)$ Let $r \in \ell_{R}\left(\bigoplus_{i \in I} X_{i}\right)$. Then by Lemma 1.1. $(r, 0) \in \operatorname{alglat}(M \oplus N)$. Thus, assuming that $M \oplus N$ is reflexive,

$$
(r, 0)=(s, s)
$$

and

$$
r=(r-s)+s \in \ell_{R}(M)+\ell_{R}(N) .
$$

Since always $\ell_{R}(M)+\ell_{R}(N) \subseteq \ell_{R}\left(\bigoplus_{i \in I} X_{i}\right)$, the proof is complete.

We note that the proof $(\Rightarrow)$ above shows that if $M \oplus N$ is reflexive (regardless of reflexivity of $M$ and $N)$, then $\ell_{R}(M)+\ell_{R}(N)=\ell_{R}\left(\bigoplus_{i \in I} X_{i}\right)$.

From the inclusions

$$
0 \subseteq \ell_{R}(M)+\ell_{R}(N) \subseteq \ell_{R}\left(\bigoplus_{i \in I} X_{i}\right) \subseteq \ell_{R}\left(X_{i}\right) \subseteq R
$$

we easily obtain the following two corollaries:

Corollary 1.3. If $M$ and $N$ are reflexive and have a common faithful subquotient, then $M \oplus N$ is reflexive.

Corollary 1.4. If $R$ is semiperfect and $M$ and $N$ are reflexive and have no common composition factor, then $M \oplus N$ is reflexive.

Proof. Suppose that $e_{1}, \ldots, e_{m}, e_{m+1}, \ldots, e_{n}$ is a complete set of primitive idempotents such that $R e_{i} / J e_{i}$ is not a composition factor of $M$ for $i=1, \ldots, m$, and $R e_{i} / J e_{i}$ is not a composition factor of $N$ for $i=m+1, \ldots, n$. Then $\ell_{R}(M)+\ell_{R}(N)=$ $R$. (See [1, Section 27].)

Regarding the Deddens-Fillmore result, the algebra generated by a matrix over a field $K$, being isomorphic to a proper factor of the polynomial ring $K[x]$, is an example of a split commutative uniserial algebra, i.e., a direct product of local uniserial rings. Since a module over a direct product of rings is reflexive precisely when its corresponding components are reflexive, to show that direct sums of reflexive modules are reflexive over such an algebra $R$, we may assume that $R$ is a local uniserial algebra, so that the ideals of $R$ are linearly ordered and every $R$-module is a direct sum of factors of $R$ (see [1, Section 32]). In this case, $R / \ell_{R}(M)$ embeds in $M$ and every subquotient of ${ }_{R} M$ is a factor of $R / \ell_{R}(M)$. In the presence of these facts Theorem 1.2 yields the following corollary almost at once. We note, however, that [10, Theorem 1] is more general. 
Corollary 1.5. The direct sum of a finite number of reflexive modules over a split uniserial algebra is reflexive.

Proof. Since we may assume that the ideals of $R$ are linearly ordered, given modules $M$ and $N$, we may also assume that $\ell_{R}(M) \subseteq \ell_{R}(N) \subseteq \ell_{R}(X)$ for every common subquotient $X$ of $M$ and $N$. So since $R / \ell_{R}(N)$ embeds in $N$, we see that both sides of the desired equality are equal to $\ell_{R}(N)$.

Next, as further applications of this theorem, we shall see that it yields particularly nice proofs of some key results in [10].

In [10], in order to show that direct sums of reflexive modules may fail to be reflexive over a split $K$-algebra $R$ with radical $J$ whose quiver contains a triple arrow, a pair of reflexive modules was constructed with diagrams (as in [8])

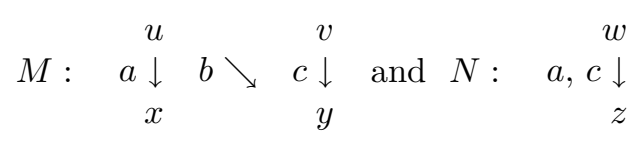

with $a, b, c$ linearly independent elements of $J \backslash J^{2}$. (The diagram indicates that $a u=x, a w=c w=z$, etc.) Here

$$
\ell_{R}(M)+\ell_{R}(N)=\ell_{R}(M)+K(a-c)+K b,
$$

but the only common subquotients of $M$ and $N$ are simple modules, so

$$
\ell_{R}\left(\bigoplus_{i \in I} X_{i}\right)=\ell_{R}(M)+J \neq \ell_{R}(M)+\ell_{R}(N) .
$$

Thus the theorem shows that $M \oplus N$ is not reflexive.

In the positive vein we shall employ the following lemma and Theorem 1.2 to obtain a simple proof of a key part of [10, Proposition 4].

Lemma 1.6. Let $R$ be a split local $K$-algebra of $\operatorname{dim}\left({ }_{K} R\right) \leq 3$. If $M$ is a faithful left $R$-module, then every proper cyclic module is isomorphic to a subquotient of $M$.

Proof. (This proof is essentially contained in the proof of [10, Proposition 4].) If $R$ is uniserial, then ${ }_{R} R$ embeds in $M$, so we may assume that $J^{2}=0$ and that $\operatorname{dim}\left({ }_{K} J\right)=2$. Let $u \in M \backslash \operatorname{Soc} M$ and suppose that $\ell_{R}(u) \neq 0$. Then, since $u \notin$ Soc $M$, there is a $b \in J$ with $\ell_{R}(u)=K b$. But then there is a $v \in M$ with $b v \neq 0$, and there is an $a \in J$ with $K a=\ell_{R}(v)$. Thus it follows that

$$
J=K a \oplus K b .
$$

Now

$$
R u \cong R / R b \text { and } R v \cong R / R a
$$

are non-isomorphic uniserial submodules of $M$ of composition length 2. If $R u \cap R v=$ 0 , we see that $\ell_{R}(u+v)=0$. Otherwise

$$
R u \cap R v=K a u=K b v=\operatorname{Soc}(R u+R v),
$$

so the annihilator of any non-zero $K$-linear combination of $u$ and $v$ is properly contained in $J$, and we may assume that $a u=b v$. Then since

$$
(k a+\ell b)\left(k^{-1} u-\ell^{-1} v\right)=a u-b v=0,
$$

we see that every proper cyclic $R$-module is a subquotient of $M$. 
Now we have the promised result of Fuller, Nicholson and Watters [10].

Proposition 1.7. Let $R$ be a split local $K$-algebra of $\operatorname{dim}\left({ }_{K} R\right) \leq 3$. If $M$ and $N$ are reflexive $R$-modules, then so is $M \oplus N$.

Proof. We may assume that $M \oplus N$ is faithful. If neither $M$ nor $N$ is faithful, then $J=K a \oplus K b$ with $a M=0$ and $b N=0$. But then

$$
\ell_{R}(M)+\ell_{R}(N)=J=\ell_{R}(R / J)
$$

and the desired equality holds. If $M$ is faithful and $N$ is not, then by Lemma 1.6 every cyclic subquotient of $N$ is a subquotient of $M$, so

$$
\ell_{R}(M)+\ell_{R}(N)=\ell_{R}(N)=\ell_{R}\left(\bigoplus_{i \in I} X_{i}\right) .
$$

Suppose both $M$ and $N$ are faithful. If $R$ embeds in $M$, then every cyclic subquotient of $N$ is a common subquotient of $M$ and $N$, so

$$
\ell_{R}(M)+\ell_{R}(N) \subseteq \ell_{R}\left(\bigoplus_{i \in I} X_{i}\right)=\ell_{R}(N)=0 .
$$

Otherwise, the cyclic subquotients of $M$ and $N$ are all proper, so by Lemma 1.6. they are all common to both modules and again

$$
\ell_{R}\left(\bigoplus_{i \in I} X_{i}\right)=0
$$

\section{Controlling}

The following the definition in [9] or [10, given bimodules ${ }_{R} M_{\Delta}$ and ${ }_{R} N_{\Delta}$, we say that $M$ controls $N$ in case for each pair $(\alpha, n)$, with $\alpha \in \operatorname{End}\left(N_{\Delta}\right)$ and $n \in N$, there is a set

$$
C_{(\alpha, n)}=\left\{\left(m_{i}, n_{i}\right) \mid i \in I\right\} \subseteq M \times N,
$$

called a connection for $\alpha$ and $n$, such that, if there are $r_{i} \in R$ with

$$
r_{i} m_{i}=0 \text { and } \alpha n_{i}=r_{i} n_{i}
$$

for all $i \in I$, then $\alpha n=0$.

Controlling is a particularly useful concept. In $[9$ it was shown that if $N$ is either generated or cogenerated by subquotients of $M$, then $M$ controls $N$; and that if $M$ is reflexive and controls $N$, then $M \oplus N$ is reflexive. Here we shall present an equivalent version of controlling that yields a partial converse to this last assertion.

Proposition 2.1. The bimodule ${ }_{R} M_{\Delta}$ controls ${ }_{R} N_{\Delta}$ if and only if, for each $0 \neq$ $\alpha \in \operatorname{End}\left(N_{\Delta}\right)$, there is a pair

$$
P_{\alpha}=\left(m_{\alpha}, n_{\alpha}\right) \in M \times N
$$

with $\alpha n_{\alpha} \notin \ell_{R}\left(m_{\alpha}\right) n_{\alpha}$ (i.e., for all $r \in R$, if $\alpha n_{\alpha}=r n_{\alpha}$, then $\left.r m_{\alpha} \neq 0\right)$.

Proof. $(\Rightarrow)$ Let $\alpha \in \operatorname{End}\left(N_{\Delta}\right)$ with $\alpha n \neq 0$ and suppose that $C_{(\alpha, n)}=\left\{\left(m_{i}, n_{i}\right) \mid i \in\right.$ $I\}$ is a connection for $\alpha$ and $n$. If $\alpha n_{i} \in \ell_{R}\left(m_{i}\right) n_{i}$ for all $i \in I$, then there are $r_{i}$ with $r_{i} m_{i}=0$ and $\alpha n_{i}=r_{i} n_{i}$ for all $i \in I$. But $\alpha n \neq 0$, so there is a pair $\left(m_{i}, n_{i}\right)$ with $\alpha n_{i} \notin \ell_{R}\left(m_{i}\right) n_{i}$. 
$(\Leftarrow)$ If $\alpha n=0$, then any $C_{(\alpha, n)}=\left\{\left(m_{i}, n_{i}\right) \mid i \in I\right\}$ is a connection for $\alpha$ and $n$. If $\alpha n \neq 0$, let

$$
C_{(\alpha, n)}=\left\{P_{\alpha}\right\}=\left\{\left(m_{\alpha}, n_{\alpha}\right)\right\} .
$$

Then it never occurs that $r_{\alpha} m_{\alpha}=0$ and $\alpha n_{\alpha}=r_{\alpha} n_{\alpha}$, so $C_{(\alpha, n)}$ is a connection.

It is worthy of note that when employing Proposition 2.1 to test for controlling, one only needs to consider those $0 \neq \alpha \in \operatorname{alglat}(N)$. Indeed, if $0 \neq \alpha \in$ $\operatorname{End}\left(N_{\Delta}\right) \backslash \operatorname{alglat}(N)$, then there is a $n_{\alpha}$ such that $\alpha n_{\alpha} \notin R n_{\alpha}$, so for any $m_{\alpha}, \alpha n_{\alpha} \notin$ $\ell_{R}\left(m_{\alpha}\right) n_{\alpha}$. Also one easily checks that $\ell_{R}(M) \subseteq \ell_{R}(N)$ whenever $M$ controls $N$.

The necessity part of the following corollary was established in [9], and has been employed in several subsequent papers. The second statement generalizes the fact that if $M$ is reflexive and controls $N$, then $M \oplus N$ is reflexive.

Corollary 2.2. The bimodule ${ }_{R} M_{\Delta}$ controls ${ }_{R} N_{\Delta}$ if and only if the restriction mapping

$$
\text { res : } \operatorname{alglat}(M \oplus N) \rightarrow \operatorname{alglat}(M)
$$

is injective.

Moreover, if these conditions hold and $\operatorname{Im}(r e s) \subseteq \lambda(R)$, then $M \oplus N$ is reflexive.

Proof. $(\Leftarrow)$ Suppose $M$ does not control $N$. Then there is an $\alpha \neq 0$ in $\operatorname{End}\left(N_{\Delta}\right)$ such that for all $m \in M$ and all $n \in N$, there is an $r \in \ell_{R}(m)$ with $\alpha n=r n$. But then $0 \neq \beta=(0, \alpha) \in \operatorname{End}\left((M \oplus N)_{\Delta}\right)$ and $\beta(m, n)=(0, \alpha n)=r(m, n)$, so $\beta \in \operatorname{alglat}(M \oplus N)$, and $\left.\beta\right|_{M}=0$.

$(\Rightarrow)$ If $\beta=(0, \alpha) \in \operatorname{alglat}(M \oplus N)$ with $\alpha \neq 0$, then for each pair $(m, n) \in M \times N$ there is an $r \in R$ with $\beta(m, n)=(r m, r n)=(0, r n)$, contrary to the condition of Proposition 2.1

For the last statement, assume that res is injective, $\delta \in \operatorname{alglat}(M \oplus N)$ and $\operatorname{res}(\delta)=\lambda_{2}(r)$ in the commutative diagram:

$$
\begin{array}{ccc}
\operatorname{alglat}(M \oplus N) & \stackrel{\text { res }}{\longrightarrow} & \operatorname{alglat}(M) \\
\lambda_{1} \nwarrow & & \lambda_{2} \nearrow \\
& R &
\end{array}
$$

Then $\operatorname{res}(\delta)=\lambda_{2}(r)=\operatorname{res}\left(\lambda_{1}(r)\right)$, so $\delta=\lambda_{1}(r)$.

Suppose that $M$ controls $N$. Then $N$ is an $R / \ell_{R}(M)$-module, and if $M$ is reflexive, then so is $M \oplus N$. Conversely we have

Corollary 2.3. If ${ }_{R} M$ is faithful and $M \oplus N$ is reflexive, then $M$ controls $N$. Thus if $M$ is a faithful reflexive $R$-module, then $M \oplus N$ is reflexive if and only if $M$ controls $N$.

Proof. As in the previous proof, we have res $\circ \lambda_{1}=\lambda_{2}$. Thus if $\lambda_{1}$ is epic and $\lambda_{2}$ is monic, then res is monic, so Corollary 2.2 applies.

A QF-3 algebra $R$ is a finite dimensional algebra with a (unique minimal faithful) module $U$ that embeds as a direct summand in every faithful module. In [9] it was shown that over a QF-2 algebra (a special type of QF-3 algebra, see [1, Section 31]) every faithful module is reflexive if $U$ is reflexive; and the problem was posed of determining whether this is the case for QF-3 algebras. Some recent progress has been made by Snashall in [15]. Perhaps this last corollary may help to shed more light on this problem. 


\section{UNIVERSAL $k$-REFLEXIVITY}

In [2] Azoff showed, from an operator theory point of view, that for an integer $k \geq 3$, if $\operatorname{dim}(\mathbb{C} M)) \leq k$, then the direct sum $M^{(k-1)}$ of $k-1$ copies of $M$ is reflexive. This topic was treated later using algebraic methods in [11]. We conclude by showing that for any left artinian ring $R$ there is an integer $k$ such that the direct sum of $k$ copies of every bimodule ${ }_{R} M_{\Delta}$ is reflexive. To do so we shall employ

Lemma 3.1. If $R / \ell_{R}(M)$ embeds in $N$ and ${ }_{R} M_{\Delta}$ controls ${ }_{R} N_{\Delta}$, then $M \oplus N$ is reflexive.

Proof. Since $R / \ell_{R}(M)$ embeds in $N$, there is an $n_{0} \in N$ with $\ell_{R}\left(n_{0}\right)=\ell_{R}(M)$. Let $\delta=(\beta, \gamma) \in \operatorname{alglat}(M \oplus N)$ and suppose that $\gamma\left(n_{0}\right)=s n_{0}$. Then for any $m \in M$, there are $r, t \in R$ with

$$
r\left(m, n_{0}\right)=\delta\left(m, n_{0}\right)=\left(\beta m, \gamma n_{0}\right)=\left(t m, s n_{0}\right),
$$

so $r-s \in \ell_{R}\left(n_{0}\right)=\ell_{R}(M)$ and

$$
\beta m=r m=s m .
$$

Thus $\operatorname{res}(\delta)=\beta=\lambda(s)$, and Corollary 2.2 applies.

Theorem 3.2. For each left artinian ring $R$ there is a positive integer $k$ such that every $R-\Delta$-bimodule is $k$-reflexive. Indeed if

$$
k=\sup \left\{c\left(\operatorname{Soc}\left({ }_{R} R / I\right)\right) \mid I \leq{ }_{R} R_{R}\right\}+1,
$$

then $M^{(k)}$ is reflexive for every bimodule ${ }_{R} M_{\Delta}$.

Proof. Let $c=c\left(\operatorname{Soc}\left({ }_{R} R / \ell_{R}(M)\right)\right)$ and let $N=M^{(c)}$ Then it is easy to see that $R / \ell_{R}(M)$ embeds in $N$ and, of course $M$ controls $N$. Thus $M \oplus N=M^{(c+1)}$ is reflexive by Lemma 3.1

This value of $k$ cannot be improved since, over the uniserial $K$-algebra $R=$ $K[x] / x^{2}$, the regular module ${ }_{R} R$ is not reflexive (see [2] or [9]).

According to [11, Corollary 3] the result of Azoff mentioned above can be extended to the $K$-algebra-bimodule case. Thus from this result and the proof of Theorem 3.2 we have

Corollary 3.3. Let $R$ and $\Delta$ be finite dimensional $K$-algebras and let $M$ be an $R-\Delta$-bimodule such that ${ }_{R} M$ is faithful. If $k \geq 2$ and

$$
\min \left\{c(\operatorname{Soc}(R R))+1, c\left(M_{\Delta}\right)-1\right\} \leq k,
$$

then $M^{(k)}$ is reflexive.

\section{REFERENCES}

[1] F.W. Anderson and K.R. Fuller, Rings and Categories of Modules, Second Edition, GTM 13 Springer-Verlag, 1992. MR 94i:16001

[2] E.A. Azoff, $k$-reflexivity in finite dimensional spaces, Duke Math. J. 40 (1973), 821-830. MR 48:9415

[3] E.A. Azoff and M. Ptak, On rank 2 linear transformations and reflexivity, J. London Math. Soc. (2) 53 (1996), 383-396. MR 97b:47003

[4] S. Brenner and M.C.R. Butler, Endomorphism rings of vector spaces and torsion free abelian groups, J. London Math. Soc. 40 (1965), 183-187. MR 30:4794]

[5] J.A. Deddens, Every isometry is reflexive, Proc. Amer. Math. Soc. 28 (1971), 509-512. MR 43:3831 
[6] J.A. Deddens and P.A. Fillmore, Reflexive linear transformations, Liner Algebra and Appl. 10 (1975), 89-93. MR 50:10856

[7] L. Ding, Separating vectors and reflexivity, Linear Algebra and Appl. 174 (1992), 37-52. MR 94a: 47075

[8] K.R. Fuller, Algebras from diagrams, J. Pure Appl. Algebra 48 (1987), 23-37. MR 89b:16018

[9] K.R. Fuller, W.K. Nicholson and J.F. Watters, Reflexive bimodules, Canadian J. Math. 41 (1989), 592-611. MR 90g:16019

[10] K.R. Fuller, W.K. Nicholson and J.F. Watters, Direct sums of reflexive modules, Linear Algebra and Appl. 239 (1996), 201-214. MR 97a:16011

[11] K.R. Fuller, W.K. Nicholson and J.F. Watters, On $k$-reflexive representations of algebras, Proc. Amer. Math. Soc. 125 (1997), 47-50. MR 97c:16027

[12] J.F. Habibi and W.H. Gustafson, Reflexive serial algebras, Linear Algebra and Appl. 99 (1988), 217-223. MR 89d:16028

[13] D. Hadwin, Algebraically reflexive linear transformations, Linear and Multilin. Alg. 14 (1983), 225-233. MR 85e:47003

[14] P.R. Halmos, Reflexice lattices of subspaces, J. London Math. Soc. 4 (1971), 257-263. MR 44:5808

[15] N. Snashall, Reflexivity of modules over QF-3 algebras, Comm. in Algebra 26 (1998), 42334242. CMP 99:05

Department of Mathematics, University of Iowa, Iowa City, Iowa 52242

E-mail address: camillo@math.uiowa.edu

Department of Mathematics, University of Iowa, Iowa City, Iowa 52242

E-mail address: kfuller@math.uiowa.edu 